\title{
Décadrages Décadrages
}

cınéma, a travers champs Cinéma, à travers champs

1-2| 2003

Le hors-champ

\section{Straub, Huillet et Cézanne}

\section{(2) OpenEdition}

1 Journals

Édition électronique

URL : http://journals.openedition.org/decadrages/600

DOI : $10.4000 /$ decadrages. 600

ISSN : 2297-5977

Éditeur

Association Décadrages

Édition imprimée

Date de publication : 1 octobre 2003

Pagination : 137-161

ISSN : 2235-7823

\section{Référence électronique}

«Straub, Huillet et Cézanne », Décadrages [En ligne], 1-2 | 2003, mis en ligne le 05 décembre 2013 ,

consulté le 22 avril 2019. URL : http://journals.openedition.org/decadrages/600 ; DOI : 10.4000/ decadrages. 600 


\section{Straub, Huillet et Cézanne}

\section{Présentation}

Sans doute l'absolu du hors-champ réside-t-il dans l'éviction même d'un film que l'absence de soutien financier condamne à l'impossibilité de voir le jour. Le dernier projet de Jean-Marie Straub et Danièle Huillet, cinéastes qui œuvrent depuis les années 60 à affirmer une démarche cohérente et anti-conformiste, en est un exemple récent.

La radicalité de leur travail, tant dans leur respect de la réalité telle qu'elle s'est offerte à la prise de vue (et de son) que dans la place centrale accordée aux textes (de Hölderlin, Kafka, Mallarmé, Vittorini,...) qu’ils ont faits leurs, semble peu compatible avec certains critères économiques de rentabilité. Procédant d'une réflexion sur la (non-)représentation au cinéma qui s'inscrit dans la filiation de la distanciation brechtienne, leurs films se montrent particulièrement exigeants envers leur public.

Après la trilogie vittorinienne (Sicilia! / Operai, contadini / Umiliati), Straub et Huillet élaborèrent un projet autour de Paul Cézanne (pour lequel ils avaient déjà témoigné leur intérêt en 1990 dans Cézanne. Conversation avec Joachim Gasquet) qui, comme à leur habitude, naquit d'une confrontation entre un texte et des images (les tableaux du peintre exposés au Louvre).

Les documents reproduits ci-dessous permettent d'une part de revenir au «découpage» initial de ce film dont le titre provisoire était Je suis Cézanne, d'autre part de rendre compte de l'accueil que lui ont réservé deux instances auprès desquelles les cinéastes ont demandé un soutien, le Louvre et Arte. Texte de présentation et découpage intégral permettront au lecteur de se faire une idée du projet et d'y éprouver la pertinence des critiques qui lui sont faites. Notons que le Musée du Louvre, qui n'est pas entré en matière en ce qui concerne la co-production du film, n'a pas non plus offert d'avantages financiers aux Straub qui tournaient dans ses murs (les Straub filmant toujours in situ).

C'est toutefois le refus d'Arte France Cinéma qui présente le plus d'intérêt, car, étant argumenté, il permet de mettre en évidence les critères de sélection qui prévalent pour de telles instances décisionnelles. Dans la réponse négative d'Arte figurait la mention d'usage selon laquelle les cinéastes pouvaient accéder, s'ils le désiraient, à la fiche de lecture de leur scénario, c'est-à-dire au document relatif à l'évaluation de leur projet. Straub et Huillet ont demandé cette "fiche» que nous 
avons annexée au dossier. Ainsi nos lecteurs pourront-ils se faire un avis sur la pertinence des jugements émis sur la base des différentes pièces de ce dossier, soit :

- la réponse du Louvre concernant la demande de co-production (page 139);

- la réponse d'Arte France Cinéma (page 140);

- la fiche de lecture d'Arte: lettre de réponse; présentation succincte; évaluation plus développée (pages 141 à 145);

- la présentation faite par Straub-Huillet de leur projet (pages 146 et 147);

- le «scénario" proposé pour Je suis Cézanne (pages 148 à 160);

- le budget global du film (page 161).

Précisons que ce film qui aurait pu hanter le hors-champ définitif du cinéma, comme tant d'autres projets qui n'ont pas su/pu/voulu se vendre, a néanmoins réintégré le champ, espace capital dans l'esthétique straubienne. En effet, en dépit du refus essuyé auprès d'Arte France Cinéma, le film a tout de même été tourné. Comme toujours chez les Straub: envers et contre tout.

Les textes qui suivent nous ont été communiqués par François Albera et sont publiés avec l'aimable autorisation de J.-M. Straub et Danièle Huillet. 


\section{Monsieur Christophe GOUGEON ATOPIC 39 , rue Durantin 75018 PARIS}

Le 13 novembre 2002
3. rue Marceau 32785 issy-Les-Moulineaux Zedex 9

rél. 0155007777 =ax. 0155007397

Monsieur,

Vous avez bien voulu nous soumettre en lecture le projet de long métrage intitulé :

\section{« JE SUIS CEZANNE »}

Ce projet a fait l'objet de lectures attentives par moi-même et nos lecteurs. Malheureusement, il n'a pas été retenu malgré ses qualités pour un examen par le Comité de Sélection d'Arte France Cinéma.

Nous recevons un nombre important de propositions. J'espère que vous comprendrez l'impossibilité dans laquelle nous sommes de sélectionner l'ensemble des projets légitimement en droit d'être co-produit par Arte.

Si vous souhaitez que l'exemplaire du scénario que vous nous avez adressé vous soit restitué, merci de bien vouloir nous le faire savoir dans un délai de quinze jours.

Nous tenons par ailleurs à votre disposition la fiche de synthèse de lecture de votre scénario, si vous le souhaitez. Dans ce cas veuillez nous en faire la demande par simple courrier.

Nous vous prions d'agréer, Monsieur, l'expression de nos sentiments les meilleurs.
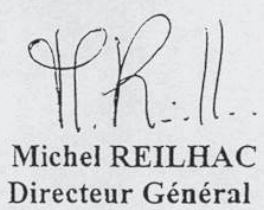


\section{Irte France Cinema}

\section{Monsieur Christophe GOUGEON \\ ATOPIC \\ 39 , rue Durantin \\ 75018 PARIS}

Le 17 décembre 2002

arte

Ref. 2002-297

3. rue Marceau

32785 issy-Les-Moulineaux

Cedex 9

-ếl. $01550077 \pi 7$

$=a x .0155007397$
Monsieur,

Pour faire suite à votre demande, veuillez trouver ci-joint la fiche de lecture de « Je suis Cézanne».

Je tiens à insister auprès de vous sur le fait qu'il ne s'agit bien évidemment que d'une opinion même si elle a contribué à forger notre décision pour ce scénario. En aucun cas la lecture de cette fiche et vos réactions, ne peuvent engager une révision de notre décision.

Cette fiche vous est transmise par souci de transparence avec vous et nous espérons que vous y trouverez une utilité.

Veuillez agréer, Monsieur, l'expression de mes meilleurs sentiments.

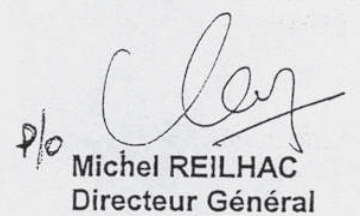


ARTE FRANCE CINÉMA

\begin{tabular}{|c|c|c|c|}
\hline CODE LECTEUR & 5 & DATE LECTURE & 05.11 .2002 \\
\hline TITRE & \multicolumn{3}{|l|}{ JE SUIS CÉZANNE } \\
\hline NATIONALITE & \multicolumn{3}{|l|}{ Française } \\
\hline RÉALISATION & $\begin{array}{l}\text { Danièle Huillet et } \\
\text { Jean-Marie Straub }\end{array}$ & SCÉNARIO & $\begin{array}{l}\text { Danièle Huillet et } \\
\text { Jean-Marie Straub }\end{array}$ \\
\hline $\begin{array}{l}\text { PRODUCTION } \\
\text { AVEC CONTACT }\end{array}$ & $\begin{array}{l}\text { Atopic (agence de } \\
\text { reportages d'idées) } \\
\text { Christophe Gougeon } \\
\text { 39, rue Durantin } \\
\text { 75018 Paris } \\
\text { Tél. : } 0142540451 \\
\text { atopic@wanadoo.fr }\end{array}$ & GENRE DU FILM & Documentaire \\
\hline LIEU DU FILM & Le Louvre & ÉPOQUE & $\begin{array}{l}\text { De nos jours / } \\
1900\end{array}$ \\
\hline SUJET & & & \\
\hline
\end{tabular}

Une visite du musée du Louvre en 1900 par Cézanne, avec la voix de Michel Piccoli.

\begin{tabular}{|l|l|}
\hline AVIS & $\begin{array}{l}\text { Danièle Huillet et Jean-Marie Straub font leur cinéma, } \\
\text { tant bien que mal, depuis de nombreuses années. Ils ont } \\
\text { leur public, restreint, mais sans doute constant, } \\
\text { puisqu'ils continuent à tourner. } \\
\text { Ils ne semblent pas se donner la peine de tendre la main } \\
\text { à leurs lecteurs, et c'est très agaçant. Ce qu'ils } \\
\text { demandent, c'est une carte blanche sur leur nom seul. } \\
\text { À titre purement personnel, cette manière de faire du } \\
\text { cinéma que je ne trouve même pas simplement } \\
\text { respectueuse, ne m'intéresse pas. }\end{array}$ \\
\hline RECOMMANDATION & NON \\
\hline
\end{tabular}

\section{SYNOPSIS}

Quelques œuvres exposées au musée du Louvre, commentées les unes après les autres par une voix, dont on apprend à la fin qu'elle était celle de Cézanne. 
Je ne suis pas sûre d'être la bonne personne pour juger de ce projet. Je n'ai jamais vu un film de Straub et Huillet, - je dois bien avouer que je n'en ai jamais eu la curiosité - et que tout ce que j'ai pu être amenée à lire sur leur cinéma n'a jamais réussi à exciter cette curiosité. Tout simplement, leur ambition de cinéma, pour autant respectable qu'elle soit, et elle l'est, depuis de si nombreuses années, ne correspond pas à la mienne - et il n'y a là de ma part aucun jugement de valeur.

À partir de là, leur nouveau projet me laisse totalement indifférente. S'il ne s'agissait pas de cinéastes connus - et reconnus -, je dois avouer que j'aurais déjà envoyé leur document aux oubliettes, sans plus de considérations, et sans scrupules.

Autant le producteur se donne la peine d'expliciter quelque peu la démarche des cinéastes, autant ce qui tient lieu de «scénario » paraît plus que succinct, et, pour tout dire, même peu respectueux des lecteurs à venir.

La forme, d'abord : écrit sur une vieille machine à écrire, qui a la méchante caractéristique de remplir tous les espaces vides (le $\langle 0 »$ est plein, mais aussi la petite boucle du «a », du « e», etc.), ce qui rend la lecture malaisée (sans compter quelques ratures), avec en plus des espaces qui ne sont pas constants... Bref, on lit «lentement», avec effort, et ce n'est pas du tout agréable. Ils n'ont pas d'ordinateur? Soit, on ne peut pas leur en vouloir. Mais leur producteur en a un, lui. Et ça aurait pris quoi ? Une heure, deux heures, peut-être, pour tout retaper proprement ? Franchement, ça en aurait valu la peine...

Le reste donne la même impression de «non-respect» du lecteur : cela aurait été si compliqué de se donner la peine de faire des photocopies couleur des tableaux commentés ? On lit des phrases qui, la plupart du temps, n'ont aucun sens pour nous puisqu'elles se rapportent à des images qu'on n'a pas sous les yeux.

Le propre d'un scénario est de «donner à voir » au lecteur le film à venir, de lui permettre de «visualiser» le film dans sa tête, grâce au travail de son imagination. Là, il aurait été si simple de « donner à voir » les tableaux, afin que le lecteur p uisse s uivre, c omme le fera le s pectateur du film, les commentaires en direct.

(NB. En faisant un effort, et sans aller jusqu'aux gros livres d'art, on arrive à trouver des reproductions d'un certain nombre des tableaux cités sur Internet: mais il faut chercher, et cela prend du temps : la production n'aurait-elle pas pu au moins nous « mâcher le travail », et nous indiquer quelques références ?) 
Donc on lit, sans vraiment d'intérêt, sans vraiment comprendre de quoi il s'agit, avec le sentiment que les auteurs n'ont pas fait les efforts nécessaires pour venir jusqu'à nous. Pourquoi nous, ferions-nous cet effort d'aller jusqu'à eux ?

D'après la fiche technique, le film est censé durer 75 minutes. Le texte que l'on a en mains ne « dure » manifestement pas ces 75 minutes. De quoi est fait le film qu'ils ont en tête? N'est-ce réellement, comme semble le suggérer ce texte, que des plans fixes de tableaux, parfois même seulement de noirs, avec une voix off?

$\mathrm{Au}$ moins, la note d'intention du producteur se donne la peine de nous donner des éléments sur Cézanne, et sous-entend que ce texte lu en voix off par Michel Piccoli est en réalité un texte de Cézanne lui-même. Mais pourquoi ne serait-ce pas de la «fiction» ? Pourquoi ne serait-ce pas les Straub et Huillet qui auraient écrit ces lignes «comme si...» elles étaient de Cézanne ? Où nous le disent-ils eux-mêmes?

Je ne suis moi-même pas vraiment familière ni avec la peinture, encore moins avec le personnage de Cézanne lui-même, pour pouvoir apprécier la « nouveauté » et la surprise de l'entendre parler ainsi.

Lisons le texte des Straub et Huillet avant la « voix off» de Cézanne : ils parlent de « construire une structure qui soit illustration, récit et matière filmique servant la matière picturale » : on peut donc supposer que le scénario n'est en effet pas encore écrit, et que la voix off n'est qu'une indication, qu'une matière brute à partir de laquelle ils veulent construire leur film.

Détail : ils parlent d'Alekan, « si la mort ne (leur) joue pas un tour » : mais Alekan est mort l'année dernière ! Ils ne se sont pas même donné la peine de relire leur texte qui semble dater au moins de plus d'un an, avant de l'envoyer en lecture?

Ils écrivent : « ainsi pourrait commencer cette aventure... », puis, « et ainsi pourrait-elle finir... », et enfin « il faut bâtir l'entre-deux, et vous pouvez nous y aider ». Cela semble sous-entendre que le scénario n'existe pas encore ? Qui est ce «rvous» auquel ils s'adressent? Les financiers ? Leur producteur?

Ensuite, ils nous donnent à lire ce texte, que l'on s uppose une v oix off (mais même cela, il faut le reconstruire), qui commence effectivement comme ils le suggéraient, se termine de même, et est organisé selon des tableaux et des noirs. Peut-on imaginer un écran noir pendant 13 lignes de texte? Comment le spectateur est-il supposé savoir que celui qui parle est Cézanne? Ne voit-on jamais un seul tableau de Cézanne lui-même? 
Bref, en l'état, on a l'impression que prendre la décision de coproduire ou non leur film revient à adhérer à leur manière de faire du cinéma, et đe leur donner carte blanche, car il est difficile de juger sur pièces leur « projet ».

C'est alors une affaire toute personnelle. En ce qui me concerne, d'un point de vue absolument subjectif, leur cinéma ne me correspond pas. Mais je respecte leur intégrité, et leur constance. Ce n'est pas un film que j'aurais envie d'aller voir, et je trouve dommage qu'ils n'aient pas plus de respect pour les partenaires auxquels ils s'adressent et dont ils ont pourtant besoin. Mais cela n'engage que moi.

Comme je suis une fille sérieuse, j'ai quand même fait quelques recherches sur Internet. Voici quelques-unes des références que j'ai trouvées, et qui peuvent aider à visualiser le film :

- la Victoire de Samothrace :

http://users.swing.be/flash_art_web/pages/La_Victoire_de Samothrace.htm - La Source, de Ingres

http://www.inter-art.com/fr/5287.htm

- L'Assassinat de Marat, par Jacques-Louis David (1748-1825)

http://www.ac-rouen.fr/colleges/fontenelle/Sitefontenelle/ipmarat.htm

- Le Paradis, du Tintoret (Jacopo Robusti, dit le Tintoret (1518-1594))

http://www.fais-

voir.com/audiovisuel/memoire/composition/script.php?num=17

\section{ASPECTS POSITIFS}

Donner à voir quelques grandes œuvres de l'histoire artistique.

\section{ASPECTS NEGATIFS}

C'est un film de Straub et Huillet, inaccessible pour la plupart des spectateurs. 
Plus janais un filn dans les musées, avions-nous juré;

trop de difficultés avec les fonctinnaires culturels, tr d'incompéhension our ce qu'ils sont censés faire aimer, la pinture, tr pe déris pour le cinéma.

Pourtant l'envie irrésistible nous a pris d'essayer de continuer, d'une autre manière bien sûr, le chemin que nous avions ouvert dans notre " Cézanne" de -989 , de revenir à la peinture et à la France aprè le théâtre, la Sieile et I'allemand a'ArmIGONE...

II nous fauàra revoir, voir mieux, voir vraiment, des toiles que rous connaissons mal, et ù Cézinne noug adera, avec sa vue perçante comrae celle de Gustav Jeonhardt dans I'ardennanee d'une partition de Bach, à découvrir les plans;

construire une structure qui soit

illustration, récit et matière filnique servant la matière pieturale (avec l'aide d'Alekan, si la mort ne nous joue pas un tour);

convaincre put-être Piccoli, par jeu

à cause de Fivette, mais aussi parce que depuis longtemps

il vulait travailler avec Straub, et que ce serait arfe

de le faire parler sans le voir - de dire "Je suis

cézanne" .

Ainsi pourrait commencer cette aventure "Une visite au Juvre vers 1900" (Alekan disait, arès le tournage de notre " Cézanne ": "V ilì Ies vrais films d'aventure") ?

"Je n'aine pas les primitifs.

Je cannais mal Giatt. Il faudrait que je le vio... Je guis tro vieux maintenant our m'en aller caurir l'Italie."

Et ainsi urrait-elle finir :

"Placez-r i ça dans son jour... C'est une infâmie, nom de Dieu! Nan, ̀̀ la fin, mais $c^{\prime}$ st vrai... Nous nous laissans toujours faire... C'est un val... I I Etat, c'est nus... I peinture... c'est mi... Qui est-ce qui emprend Courbet?... On le 
fout en prison dans cette cave... Je preteste... J'irai trouver les journaux, Vailès... Qu'on fasse porter cetta toile à sa lace, dans la lumière... Qu'on la vie... Nus avens en France une machine pareille, et nous la cachons... Qu'on foute le feu au rouvre, alors... tout de suite... Si n a peur de ce qui est beau... Je suis céranne."

Il faut bâtir I'entre-deux, et vous puvez nous y aider. 
1. PANO (du pont)

dedroite à gauche

2. NOIR

Je n'aime pas les primitifs.

Je connais mal Giott. Il faudrait que je le voie. Je suis trop vieux maintenant pour m'en aller courir l'Italie.

Je n'entre presque jamais dans la salle des primitifs. Ce n'est pas de la peinture pour moi... que voulez-vous que me fassent les maładresses de Cimabue, les nalvetés de l'Angelico et même les perspectives d'Uccell... Il n'y a pas de chair sur ces idées.

3. IA VICTOIRE DE SAMOTHRACE

Tenez. Regardez-mi ça... C'est une idée, c'est tout un peuple, un monent héroique dans la vie d'un peuple, mais les éteffes collent, les alles battent, les seins se gonflent. Je $n^{\prime} a i$ pas besoin de voir la tête pour inaginer le regard, parce que tout le sang qui fouette, circule, chante dans les janbes, les hanches, tout le eorps, il a passé en torrent dans Ie cerveau, il est monté au coeur. Il est en mouvement, il est le mouvement de toute la femme, de toute la statue, de toute la Grèze. Quand la tête s'est détachée, allez, le marbre a saigné...

4. NOIR

Tandis que là-haut, vous pouvez avec le sabre du bourreau, cauper le cou ̀े tous.ces petits martyrs. Un peu de vermilion, des gouttes de sang, ça... Ils sont déjà tout envolés en Dieu, exsangues. On ne peint, pas des âmes. Et tenez, les ailes dela victoire, on ne les vit pas, je ne les vis plus. on n'y pense plus, tant elles apparaissent naturelles. Je corps n'a pas besoin d'elles pour s'envoler en plein triomphe. Il a sen élan... Tandis que les auréles, wutour du christ, des vierges et des Saints, on n'aperģoit qu'elles. Elles s'imposent. Elles me gênent. Que voulez-vous. On re peint pas des ames. On peint des corps; et quand les corps sont vien peints, foutre! l'âme, s'ils en avaient une, l'âme de toutes parts rayonne et transparast. 
5. IA SOURCE

Ingres non plus, parbleu, $n^{\prime} a$ pas de sang. Il dessine. Iues primitifs dessinaient. Ils cloriaient, ils faisaient, en grana, du coloriage de missel. Ia peinture, ce qui s'appelle Ia peinture, ne nait qu'avec les Vénitiens... Oh! c'est très beau Ingres, Raphæel et toute la boutique.Je ne suis pas plus bouché qu'un autre. J'ai le plaisir de la ligne, quand je veux. Mais ii y a lì un écueil, Holbein, clouet ou Ingres n'ont que la ligne Eh! bien, ça ne suffit pas. C'est très beau, mais ça ne suffit pas. Regardez cette Source... C'est pur, c'est tendre, c'est suave, mais... gia ne tourne pas dans l'air. Ie rocher de carton n'échange rien ảe son humidité pierreuse avec le marbre de cette chair mouillée... ou qui devrait l'être. Où y a-t-il pénétration ambiante? Et puisqu'elle est la source, elle devrait sortir de l'eau, du rocher, des feuilles; elle est collée contre. A force de vouloir peinare la vierge idéale, il n'a plus peint un corps du tout.

... Jar esprit de système. Système et esprit faux.

6. NOIR

David a tué 1 a peinture. Ils ont introduit le poncif. Ils ont voulu peindre le pied idéal, la main idéale, le visage, le ventre parfaits, I'être suprême. Ils ont hanni le caractère. Ce qui fait le grard peintre, c'est la caractère qu'il donne à tout ce qu'il touche, la saillie, le mouvement, la passion, car il y a des sérénités passionnelles. Eux en ont peur, ou plutôt ils n'y ont pas songé. Par réaction peut-être contre la passion, les tempêtes la brutilité søciale de leur époque.

\section{L'ASSASSINAT DE HARAT}

...Je ne connais rien de plus frøid que son marat! Quel héros étriqué! Un homme quiarvait été son ami, qui avait été assassiné, qu'il nevait glorifier aux yeux de Paris, de la Franee entière, de toute 12 postérité. L'a-t-il assez rapetassé avec son drap et délavé dans sa baignoire. Il pensait à ce qu'on dirait du peintre et non à ce qu'on penserait de Marat. Mauvais peintre. 
8. NOIR

Et il avait eu le cadavre devant les yeur... Tenez, quelque chose d'immonde, ce sant ses caricatures. Illes m’ont révélé, du coup, toute la mécanique grinçante de cet esprit. ...II est le dernier peut-être qui ait su son métier mais qu'en a-t-il l'ait bon Dieu? Ies boutons de culotte de sa Remise des aigles.

\section{IAA REMISE DES AIGLES}

Alors qu'en grande pompe, il aurait dû nous donner, ̀े la Titien, la psychologie detous ces palefreniers et de tous ces goujats autour de leur crapule couronnée. Sale Jacobin, sale classique... vous savez, dans les origines, ee que raconte Taine de l'esprit elassique! Ah! David en est bien le terrible exemple. ce vertueux:

10. NOIR

Il est arrivé ł̀ châtrer, dans sen art, mêne ce paillard d'Ingres, qui adorait la femelle pourtant...

11. IES NOCES DE CAMA, Ensemble

Mais voilà de la peinture. Voilà de la peinture. Te morceau, l'ensemble, les valumes, les valeurs, la composition, le frisson, tout y est... icoutez un peu, c'est épatant!... Qu'est-ce que nous somes?... Fermez les yeux, attendez, ne pensez plus a rien. Ouvrez-les... N'est-ee pas?... On ne perçit qu'une grande ondulation colorée, hein? une irisation, des couleurs, une richesse de couleurs. C'est ģa que doit nous donner d'abord le tableau, une chaleur harmonieusé, un abine où l'øeil s'enfonce une sourde germination. In état de grace colorée. Tous ces tons vous coulent dans le' sang, n'est-ce pas? On se sent ravigoté. On nait au monde vrai. On devient soi-même, on devient de la peinture... Pour aimer un tableau, il faut d'abord l'avoir bu ainsi, à longs traits. Perdre conscience. Descendre avec le peintre aux racines sombres, enchevêtrées, des choses, en revionter avec les couleurs, s'épanouir à la lumière avec elles Savoir voir. Sentir... Celui-lì, allez, il était heureux. Et tous ceux qui le comprennent, il les rend heureux... Ies choses, les êtres lui entraient dens l'âme avec le soleil, sans rien qui les lui sépare de la lumière, sans dessin, sans abstractions, tout en couleurs. Ils en sortaient, un jour, les mêmes, mais on ne sait pourquoi, habillés d'une gloire douce. Tout heureux comne s'ils avilent respiré une mystérieuse musique. 
12. IES NOCES DE CAINA, Détail

Celle qui rayonne, voyez, de ce groupe, au milieu, que les femmes et ies chiens écoutent, que les hommes caressent avec leurs fortes mains. Ia lénitude de la pensée dans le plaisir et du plaisir dans la santé, écoutez un peu, je crøis que c'est Féronèse, la plénitude de I'idée dans les eøuleurs. Il couvrait ses toiles d'une vaste grisaille, oui, comme ils faisaient tous à cette époque, et c'était sa première emprise, comme un morceau de la terre avant que le jour, l'esprit se lève...

\section{IES TOCES DE CANA, Ensemble}

Ie dessous, les dessous. Je disais bien. Il préparait, d'une immense grisaille... I'idée décharnée, anatomique, squelettique de son univers, la charpente douce qu'il lui fallait, et qu'il allait habiller de nuanees, avec ses couleurs et ses glacis, en tassant les mores. Un grand monde pâle, ébäuché, encore dans les limbes. Il me semble que je le vois, tenez, entre le tissu de la toile et la chaleur prismatique du soleil... On empâte tout de suite aujourd'hui, on attaque grossièrement comme un maçon, et l'on se croit très fort, très sincère... Va te faire fiche. On a perdu cette seience des préparations, cette vigueur fluide que donnent les dessous. Moduler, non, moduler. Il faut moduler... Aujourd'hui! on revient, on gratte, on regratte, on épaissit. C'est un mortier. ou bien, les plus sormaires, vous savez, ils cernent brutalement leurs bonshommes, leurs - bjets, d'un trait brut, schématique, appuyé, et en teintes plates on remplit jusqu'au bork. C'est criard comme une affiche, neint comme au pochoir, ̀̀ l'emporte-pièce. Rien ne vit.

\section{IES NOCES DE CANAA, Détail}

Tandis que voyez-vous cette robe, cette femme, eette créature, contre cetie nappe, ì commence sur son sourire l'ombre, où la lumière caresse-t-elle, boit-elle, imbibe-t-elle cette - mbre on ne sait pas. Tous les tons se pénètrent, tous les volumes tournent en s'ambostant. Il y a continuité... Je ne nie pas que des fois, sur nature, il n'y ait de ces effets brusques d'ombre et de lumière, par bandes vilentes, mais ee n'est pas intéressant. Surtout, si ça devient un procédé. 


\section{IES NOCES DE CANA, Ensemble}

Ie magnifique, e'est de baigner toute une composition infinie conme celle-ci, immense, de la même clarté atténuée et chaude et de donner à l'oeil l'impression vivante que toutes ces poitrines respirent véritablement, mais là come vous et moi, l'air doré qui les inonde. Au fond, j'en suis sûr, ce sont les dessous, l'ame secrète des dessous, qui, tenant tout lié, donnent cette force et cette légèreté ̀̀ l'ensemble. Il faut commencer neutre. Apròs, il pouvait s'en donner à coeur-joie, emprenez-vous? Sacristi! le chic parfait, le chic exquis, I'audacieux de tous les ramages, des étoffes qui se répondent, des arabesques qui s'enlacent, des gestes qui se continuent. Est-ce assez ça? non, mais est-ce assez gà? Tous pouvez détailler. Tout le reste du tableau vous suivra toujours, sera toujours là présent. Vous sentirez la rumeur autour de la tête, du morceau que vous étudierez. Vous ne pouvez rien arracher à l'ensemble... Ce n'étaient pas des peintres de moreeaux, ceux-la emme nous... Devant ça, quelque chose chez les modernes flanche Quei?... Dites, quoi?... Nous allons voir. Nous allons voir. Tenez, prenez à gauche, là, partez de eette colonne, est-elle de marbre, sacrédié! et lentement, des yeur, faites tout le tour de la table... Est-ce beau? Es $\tau-c e$ vivant?... Et en même temps, est-ce transfigué, triomphant, miraculeux, dans un monde autre et cependant tout réel. Ie miracie y est, l'eau changée en vin, le monde changé en peintare. On nage dans la vérité de la peinture...

Dire que j'ai voulu brûler ça, cans le temps. Par manie d'originalité, d'inventer... Quand on ne sait pas, on croit que ce sont ceux qui savent qui vous obstruent. Alors qu'au contraire si on les fréquente, au lieu de vous encombrer, ils vous prennent par la main, et vous font gentinent, à côté d'eux, balbutier votre petite histoire. 
16. IA FIILE DE JATRE ("JÉsus chez le Pharisien")

Ga, par exemple, c'est peut-Être encore plus épatant... Cette gamme d'argent... Tout le prisme qui se fond dans le blanc. Et ce que j'aime, vous savez, dans tous ces tableaur de véronèse, c'est qu'il n'y a pas à tartiner sur eux. On les aime, si on aime lá peinture. On ne les aime pas, si on cherche de la littérature la côté, si on s'excite sur I'anecdote, le sujet Un tableau ne représente rien, ne doit rien représenter d'abord que des couleurs... Lioi, je déteste ça, toutes ces histoires, cette psychologie, ces peladaneries autour. Parbleu, ça y est dans la toile, les peintres ne sont pas des imbéciles, mais il faut le voir avec les yeux, avec les yeux, vous m'entendez bien. Ie peintre n'a pas voulu autre chose. Sa psychologie, c'est la rencontre de ses deux tons. Sin émotion est là. c'est àa son nistoire, sa vérité, sa profondeur, à lui. Puisou'il est peintre, voyons! Et ri poète ni philosophe.

... On raconte que la nuit de la naissance du sauveur les vignes fleurirent dans toute la Palestine. Nous, peintres, nous devons plutêt peindre la floraison de es vignes que les tourbilions d'anges qui trompettent le Messie. He peignons que ce que nous avons vu, ou ee que nous pourrions voir... Comme 1ai, tenez.

\section{CONCERT CHAIPÊTRE}

Embellissons, ennoblissans d'un grand rêve charnel toutes nos imaginations... II I is baignons-les dans la nature. Ne tirans pas la nature à elles. Tant pis, si nous ne pouvons pas. rous comprenez, il aurait fallu, dans le déjeurer sur I'herbe, que Manet ajoute, je ne sais pas, moi, un frisson de cette noblesse, on ne sait quoi qui, ici, emparadise tous les sens. Voyez la coulée d'or, da la grande femme, le dos de l'autre... Elles sont vivantes, et elles sont divines. Tout le paysage dans sa rousseur est comrne une églogue surnaturelis, un moment balancé de l'univers dans son éternité perçue, dans sa joie plus humaine. Et on y participe, on ne méprise rien de sa vie. 
18. IA CUISINIE DES ANGES

C'est comme là... Il y a là une nature morte prodigieuse. Hurillo a dû peindre des anges, mais quels éphèbes, voyez comme leurs pieds nerveux posent bien sur la dalle. Vraiment ils sont dignes d'éplucher ces bedux légumes, ces carottes et ees chøux, de se mirer dans ces ehaudrons... Ie tableau lui était comandé, n'est-ce pas?... Il s'est laissé aller, pour une fois. Il a vu la seène... Il a vu des êtres radieux entrer dans cette cuisine de couvent, de jeunes charretiers eélestes, la beauté de la jeunesse, la santé éclatante, chez tous ces mustiques, ces épuisés, ces tøurmentés. Vøyez comne il ppose la maigreur jaunâtre, l'extase hystérique du saint en prière aus gestes ealmes, à la cerititude rayonnante de tous ces beaux ouvriers. Et le tas de légumes! on peut passer des navets et des assiettes aux ailes sans changer d'air. Tout est réel... 
Tintoret, voilà le peintre. Comme Reethoven est le musicien, Platon le philosophe...

J'ai f'euilleié tout ce que j'ai pu trouver de son oeuvre. Elle est gigantesque. Tout y est, de la nature morte à Dieu. C'est l'arche immense. Toutes les formes d'existence, et dans un pathétique, une passion, une invention incroyables. Si j'étais allé à Vonise, e'eut été pour lui. Il parât qu'an ne le connât, que 1 à...

Chaste et sensuel, brutal et cérébral, volontaire autant qu'inspiré, sauf la sentinentalité, je erois qu'il a tout connu, ce Tintoret, de ce qui fait la joie et le tourment des hommes... Eevutez un peu, je ne puis pas en parler sans trembler... Ses portraits, terribles, me l'ont rendu familier... Celui que lanet a copié...

-- On dirait un Cézanne.

Ah! je voudrais bien... Vous savez, il me semble que je I'ai eonnu. Je le vois, rompu de travail, harassé de couleurs, dans cette chambre tendue de pourpre de son petit palais, comme moi dans mon catouchon du Jas-de-Bouffan, mais, iui, toujours, même en plein jour, éelairé d'une lampe fumeuse, avec l'esnèce de théâtre ì marionnettes ù il préparait ses grandes compositions. Hein... ce guignol épique!

Quand il quittait ses chevalets, paraft-il, il arrivait là, il tombait, épuisé, toujours farouche, e'était un grognon dévoré de aésirs sacrilèges... Oui, ui... il y a un drame terrible duns sa vie... Je n'ose pas le dire... Suant à grosses gouttes, il se faisait endormir par sa fille, il se faisait jouer du violoncelle par sa fille, des heures. Seul avec elle, dans tous ces reflets rouges... Il s'enfonçait dans ce monde enflammé, - ì la fumée du notre s'évanouit... Je le vois... Je le vois... Ia lumière se dépouillait du mal... Eł vers la fin de sa vie, lui, dont la palette rivalisait avee l'arc-en-ciel, il disait ne plus chérir que le noir et le blane... Sa fille était morte.. le noir et le blanc!... Parce que les couleurs sant méchantes, torturent, comprenez-vous... Je connais eette nostalgie.

Est-ce qu'on sait? on cherche une paix définitive... Ce paradis. Allez, pour peindre cette rose de joie, tourbillonnante, il faut avoir beaucoup souffert... beaucoup souffert, je vous en fiche monbillet. Nهus sommes à l'autre pêle, ici. Iuà-bas, ce beau prince de Véronèse. Ici, ce forçat de Tintoret. Cet espèce de misérable qui a tout aimé, mais dont un feu, une fièvre consunait tous les désirs ausitêt qu'ils naiggaient. Voyez son ciel... Ses bons dieux tournent, tournent. Ils n'ont pas le paradie calme. C'est une tempête, ce repes. Ils continuent l'élan qui, comme lui, les a dévorés, toute leur vie. Ils en jouissent maintenant, après en avoir tant souffert. J'aime ça... 
20. IE PARADIS, Détail

It voyez ce pied blanc, ici, à gauche... Les dessous, encore... il préparait ses chairs en blanc. Puis d'un glacis rouge, vlan, voyez à coté, il leur dornait la vie. Blanc et noir, je ne peux plus peindre qu'en biane et noir, eriait-il à la fin. Comnent aurait-il fait? Comment se serait-il passé de son supplice? on peut tout attendre d'un tel honhomme. Dans sa jeunesse, il avait eu le culot d'affirmer: 12 colleur de Titien das le dessin de Michel-Ange. Dt il y est arrivé. Titien l'avait flanqué à la porte...

\section{PIAN FIXE - de la Salle des Etats}

Au fond, celui qui rendrait ça, simplement, la seine, Paris, un jour de Paris, pourrait entrer ici, la tête haute...II faut être un bon ouvrier. N'être qu'un peintre.

22. IE TRIOWPHE D'HOMERE

... L'orangé pour dire la colère d'Achille et les flammes de Troie, le vert pour dire les voyages d'Ulysse et les remous de l'océan... hiais ce n'est pas ça, la formule! Oui, oui, la formule qui vous étraint...

...Ici, ils ne sont que deux: Delacroix et Courbet. Ie reste, c'est de là fripuille... 


\section{FEMIIES D'ALGER}

Nous y sommes tous dans ce Delacroix. Puand je vous parle de la joie des couleurs pour les couleurs, tenez, c'est cela que je veux dire... Ces roses pales, ces coussins bourrus, cette babouche, toute cette limpidité, je-ne sais pas moi, vous entre dans I'oeil comme un verre de vin dans le gosier, et on est tout de suite ivre. On ne sait comment, mais on se sent plus léger. Ces nuances ailègent et purifient. Si j'avais commis une mauvaise action, il me semble que je viendrais là-devant pour rie remettre d'aplomb... Et c'est bourré. Ies tons entrent les uns dans les autres, comme des soies. Tout est cousu, travaillé d'ensemble. Et c'est pour ça que ça tourne. C'est la première fois qu'on a peint un volume, depuis les grands. It chez Delacroix, il n'y a pas à dire, il y a quelque chose, une fièvre qui n'est pas chez les aneiens. C'est la fièvre heureuse de la convalescence, je crois. Avec lui, la peinture sort du marasme, de la maladie des polonais. Il bouscule David. Il peint par irisation.

Et puis, il est convaincu que le soleil existe et qu'on peut y trerper ses pinceaux, $y$ faire sa lessive. Il sait différencier. ce n'est plus comme chez Ingres, İ̀-bas, et tous ceux qui sont ici... Une soie est un tissu et un visage de la chair. Ie même soleil, la mêne émotion caresse, mais se aiversifie. Il sait sur le flanc de cette négresse accrocher une étoffe qui n'a pas la nême odeur que la culotte parfumée de cette géorgienne, et c'est dans les tons qu'il sait ga et qu'il le met. Il contraste. Toutes ces nuances poivrées, voyez, avec toute leur vidlence, la claire harmonie qu'elles donnent. It il a un sens de l'être humain, de la vie en mouvement, de la tiéceur. Tout bouge, tout chatoie. Ia lufière!... Dans son intérieur il y a plus de lunière chaude que dans tous ces paysages de corot et ces batailles d'à côté. Regardez. Son ombre est colorée. II nacre ses dégradés, ce qui assouplit tout... 
24. L'ENTPEE DES CFOISESS

Son Intrée des Croisés, c'est terrible... autant dire que vous ne la voyez pas. Nous ne la vojons plus. J'ai vu, moi qui vous parle, mourir, pâlir, s'en aller ce tableau. e'est à pleurer. De dix ans en dix ans, il s'en va... Il n'en restera, un jour, plus rien... Si vous aviez vu la mer verte, le ciel vert. Intense Et comme les fumées étaient plus dramatiques alors, les navires qui braient, et comme tout le groupe des cavaliers se présentait. Quand il l'exposa, on cria que le cheval, ce cheval, était rose. C'était magnifique. Une rutilence. Mais ces sacrés romantiques, avec leur dédain, usaient d'atroces matières. Ies droguistes les ont volés comme dans un bois. C'est comme le laufrage de Géricault,

25. IEE NAUFRAGE

une page superbe, on ne voit plus rien.

26. I'ENTREE DES CROISÉS

Ici, il reste encore la mélancolie rongée des visages,

I. tristesse de ces éperonnés, mais tout cela, nous nous en souvenons, c'était dans les teintes de Delacroix, et les fonds s'étant éteints, son effet, son âme n'y est plus. Je les ai encore vus, moi, ces couronnes livides. Ils ne s'avancent plus dans le flamboiement, dans cet air d'orient...

Voilà, tenez, ce qui ppouve mieur que tout que Delacroix est un vrai peintre, un bougre de grand peintre. Ce n'est pas I'anecdote des Croisés, ils étaient anthropophages, dit-on, ce n'est pas leur humanité apparente, c'était le tragique de sestons qui faisaint son tableau et qui exprimait toute l'âme pourrie de ces mornes vainqueurs. Alors la belle grecque mourante la femme de soie abandonnée dans ses riches atours, la barbe du vieillard, les chevaux carapaçonnés et les hampes Iugubres, dans les fusions chantantes, prenaient tout leur sens.

Ça mourait, pleurait et reniflait. Dans les couleurs. ifaintenant il ne reste plus qu'une inage... Ies rermes d'Alger, elles, n'ont pas bougé.

27. FEHIHES D'ALGEP

Ii'Entrée était peinte aussi éclatante. ...Delacroix, c'est le romantisme peut-être. Il s'est trop gorgé de Shakespeare et de Dante, il a trop feuilleté paust. Il reste la plus belle palette de France, et personne, sous notre ciel, écoutez un peu, n'a eu plus que lui le charme et le pathétique ì la fois, la vibration de la couleur.

- Et Cøurbet? 
Un batisseur. Un rude gacheur de platre. Un broyeur de tons. Il maçonnait comme un romain. It lui aussi, un vrai peintre. Il n'y en a pas un autre dans ce siècle qui le dégote. Et il a beau retrousser sas manches, se coller le feutre sur l'oreille, déboulonner la colonne, sa facture est d'un classique! sous ses grands airs fendants... Il est profond, serein, velouté. Il y a de iui des nus, dorés comme une moisson, dont je raffole. Sa palette sent le blé... Oui, oui, Proudhon lui a tourné la tête avec son réalisme, mais au fond, ce faneux réalisme, c'est comme le romantisme de Delacroix, il ne le faisait, vaille que vaille, rentrer à grands coups de brosses que dans quelques toiles, ses plus tapageuses, les moins belles sârement. Et encore, il était plus dans ie sujet, son réalisne, allez, que dans le métier. Il voit toujours composé. Sa vision est restée celle des vieux. C'est comme du couteau, il ne s'en servait que dans le paysage. C'est un raffiné, un fignoleur. Vous savez le mot de Decamps. Courbet est un malin. Il fait de la peinture grossière, mais 11 met le fin par dessus. Et moi, je dis que c'est la force, le génie, qu'il mettait par dessous... Il a beau faire large, il est subtil.

-- Courbet est la grand peintre du peuple.

Et de la nature. Son grand apport, c'est l'entrée lyrique de la nature, de l'odeur des feuilles mouillées, des parois moussues de la forêt, dans $1 a$ peinture cu dix-neuvième siècle, le murmure des pluies, l'ombre des bois, la narche du soleil sous les arbres Ha mer. Et la neige, il a peint la neige comme personre! ...ces grands paysages blancs, plats, sous le crépuscule grisatre sansungésérité, tout ouatés... Formidable, un silence a'hiver... Et le couchant, la bande sanguinolente, la mare, l'arbre qui fuit avec la bête, dans les yeux de la bête... Tous ces lacs savoyards avec le clapoternent de l'eau, la brume qui monte des rives, enveloppe les montagnes... Ía grande vague, avec son enchevêtrement écumeux, sa marée qui vient du fond des âges, tout son ciel loqueteux et son âpreté livide. 


\section{I' ENTERREWENT}

on a dit qu'il a peint ģa aprés 1 a mort de sa mère. Il s'était enfermé un an à Ornuns. Ce sont les gens du village qui lui posaient, sans poser. Il les avait dans l'oeil... Dans une espèce de grenier... IIs venaient se reconnaftre... Il mêlait ees grotesques à sa douleur... Flaubert... iliais on a dit ça. Ia légende est pius forte que l'histoire. Sa mère n'était pas morte. Blle a posé, elle st dans un coin... mais pour dire combien la machine est émue. Elle recrée, elle imagine la vie. Oui, comme flaubert a tiré le roman de Falzac, Courbet, peut-être de l'empartement romantique, de la véraeité expressive de Delacroix, a tiré... vous rappelez-vous dans ce voyage qu'il fait le père Flaubert, dans par les champs et par les grèves, cetenterrement qu'il raconte et cette vieille qui pleure corme il pleut... Chaque fois que je le relis, je pense à courbet... I a même émotion... Sacrérié! que e'est beau... voyez ce chien... Velasquez! Velasquez! Ie chien de Philippe est moins chien, tout chien de roi qu'il était... Yous l'avez vu... Et l'enfant de choeur, ce rouge joufflu... Renoir peut y venir...

... II n'y a que courbet qui sache plaquer un noir, sars trouer la toile... Il n'y a que lui...Ici, comme dans ses rochers et ses troncs, là-bas. Il pouvait, d'une coulée descendre tout un pan de vie, l'existence minable d'un de ces gueux, voyez, et il revenait ensuite, avec pitié, par bonhommie de doux géant qui comprend tout... I. caricature se trempe de larmes... ... Qui est-ce qui comprend Courbet? on le fout en prison dans cette cave... Je proteste... J'irai trouver les journaux, valiès. Qu'on fasse porter cette toile à sa place, dans la lumière... Qu'on la voie... Nous avons en France une machine pareille, et nous la cachons... Qu'on foute le feu au I,ouvre, alors... tout de suite... Si on a peur de ce qui est beau... Je suis Cézanne.

30. PANO ( $\mathrm{du}$ Pont)

de la gauche vers la droite

- Notre Iame 


\section{JE SUIS CEZANNE}

durée : $75^{\prime}$ format : $35 \mathrm{~mm}$

Une visite au Louvre vers 1900

Danièle Huillet, Jean-Marie Straub image : William Lubtchansky voix : Michel Piccoli ou Julie Koltai

\section{BUDGET}

\section{récapitulatif}

droits artistiques

personnels

charges sociales

moyens techniques

transports, défraiements, régie

post-production

assurances

en Euros

total

28500

76500

46185

34030

4855

81300

3800

275170

imprévus

$10 \%$

frais généraux

19260

frais financiers

total général 